Timmer e.a.

\title{
Dopaminergic drugs decrease loss aversion in Parkinson's disease with but not without depression
}

\author{
Monique H.M. Timmer ${ }^{1,2}$, Guillaume Sescousse ${ }^{1}$, Rianne A.J. Esselink ${ }^{1,2}$, Payam Piray ${ }^{1}$, \\ Roshan Cools ${ }^{1,3}$
}

${ }^{1}$ Donders Institute for Brain, Cognition and Behavior, Centre for Cognitive Neuroimaging, Radboud University, 6500 HB Nijmegen, the Netherlands

${ }^{2}$ Radboud University Medical Centre, Department of Neurology and Parkinson Centre Nijmegen (ParC), $6500 \mathrm{HB}$ Nijmegen, the Netherlands

${ }^{3}$ Radboud University Medical Centre, Department of Psychiatry, 6500 HB Nijmegen, the Netherlands

Corresponding author: Monique H.M. Timmer, Radboud University Medical Centre, Department of Neurology (HP 935), PO Box 9101, 6500 HB Nijmegen, the Netherlands. Phone: +31-24-3615091; Fax: +31-24-3541122; E-mail: Monique.Timmer@radboudumc.nl.

Short title: Loss aversion in Parkinson's disease

Number of pages: 31

Number of: Figures 4; Tables 2

Total words: Abstract 185; Introduction 573; Discussion 968

\section{Conflict of Interest}

The authors declare no competing financial interests.

\section{Acknowledgements}

This project was funded by a grant from the "Stichting Parkinson Fonds", Hoofddorp, the Netherlands. We would like to thank all participants for their cooperation in the study. 
Timmer e.a.

1

2

3

4

5

6

7

8

9

10

11

12

13

14

15

16

\section{Significance statement}

18

19

20

21

22

23

24

\section{Abstract}

Depression, a common non-motor symptom of Parkinson's disease (PD), is accompanied by impaired decision making and an enhanced response to aversive outcomes. Current strategies to treat depression in PD include dopaminergic medication. However, their use can be accompanied by detrimental side effects, such as enhanced risky choice. The mechanisms underlying dopamine-induced increases in risky choice are unclear. In the current study we adopt a clinical-neuroeconomic approach to investigate the effects of dopaminergic medication on loss aversion during risky choice in depressed and non-depressed PD. Twenty-three healthy controls, 21 depressed and 22 non-depressed PD patients were assessed using a well-established gambling task measuring loss aversion during risky choice. Patients were tested on two occasions, after taking their normal dopaminergic medication (ON) and after withdrawal of their medication (OFF). Dopaminergic medication decreased loss aversion to a greater extent in depressed than non-depressed PD patients. Moreover, we show that the degree to which dopaminergic medication decreases loss aversion correlated with current depression severity and with drug effects on depression scores. These findings demonstrate that dopamine-induced changes in loss aversion depend on the presence of depressive symptoms in PD.

Dopaminergic medication that is used to treat motor and non-motor symptoms in patients with Parkinson's disease is known to contribute to risky decision-making. The underlying mechanisms are unclear. The present study demonstrates that dopaminergic medication in Parkinson's disease decreases loss aversion during risky choice, but only in depressed and not in non-depressed patients with Parkinson's disease. These results advance our understanding of the mechanisms underlying dopamine-induced risky choice, while also identifying depression as an important factor that confers vulnerability to such dopamine-induced risky choice. 
Timmer e.a.

\section{Introduction}

Depression is a common non-motor symptom of Parkinson's disease (PD) which greatly affects quality of life (Schrag, 2006). Similar to the motor symptoms, depression in PD can be treated with dopaminergic medication (Barone et al., 2010; Stacy et al., 2010; Seppi et al., 2011). However, their use is limited by potential side effects, such as enhanced risk-taking behavior, in their most severe form qualifying as impulse control disorder (ICD) (Weintraub et al., 2010; Voon et al., 2011b). The mechanisms underlying dopamine-induced increases in risky choice have remained unclear.

One mechanism by which dopaminergic medication can increase risky choice is by attenuating loss aversion. Loss aversion reflects the relative weighting of gains and losses during risky choice and is one of the core concepts of Prospect Theory, a well-known economic theory of decision-making under risk (Kahneman and Tversky, 1979, 1984). In the domain of learning, dopamine manipulation studies in healthy controls and PD patients have revealed that the balance between learning from reward and punishment critically depends on striatal dopamine (Frank et al., 2004; Cools et al., 2006; van der Schaaf et al., 2014). Increases in dopamine enhance reward-based learning/choice while impairing punishmentbased learning/choice and decreases in dopamine enhance punishment-based learning/choice while impairing reward-based learning/choice. One obvious next question is whether dopaminergic medication alters risky choice in an analogous manner, by increasing the weighting of prospective rewards (gains) relative to punishments (losses). This relative weighting corresponds exactly to loss aversion in the context of Prospect Theory.

Depression has been associated with reduced reward and enhanced punishment sensitivity across various domains including decision making (Eshel and Roiser, 2010). For instance, depressed individuals (without PD) have been shown to exhibit reduced reward-based reversal learning and attenuation of associated BOLD signal in the ventral striatum (Robinson et al., 2011). Depressed patients also exhibit 
Timmer e.a.

enhanced loss-minimization and attenuated gain-maximization as well as enhanced loss aversion during risky choice (Gradin et al., 2011; Maddox et al., 2012; Chandrasekhar Pammi et al., 2015). This cognitive profile, together with clinical observations that depressive symptoms in PD occur more often during OFF

51 periods (Maricle et al., 1998) and can (to some degree) be alleviated by dopaminergic medication

52 (Barone et al., 2010), concurs with evidence indicating that depression in PD is associated with dopamine deficiency in the ventral striatum (Weintraub et al., 2005; Vriend et al., 2013; Vriend et al., 54 2014).

Based on current available evidence, we put forward two opposite hypotheses about the effects of dopaminergic medication on loss aversion in depressed PD patients. According to one account of drug-

57 induced cognitive deficits, the "dopamine overdose hypothesis", dopaminergic doses necessary to remedy the severely dopamine depleted dorsal striatum and associated cognitive and motor functions, might detrimentally overdose relatively intact ventral striatal dopamine levels (Gotham et al., 1988; Swainson et al., 2000; Cools et al., 2001). One implication of this hypothesis is that drug-induced increases in risky choice are restricted to patients with intact ventral striatal dopamine levels, while not extending to patients with already depleted ventral striatal dopamine levels, such as those with comorbid depression. The alternative hypothesis stems from clinical observations showing that specifically depressed PD patients are more likely to experience ICDs (Isaias et al., 2008; Joutsa et al., 2012), suggesting that dopamine-induced increases in risky choice are restricted to depressed patients. task measuring loss aversion on two occasions: once ON and once OFF dopaminergic medication.

\section{Materials \& Methods}


Timmer e.a.

\section{Participants and experimental design}

71 We recruited 23 non-depressed PD patients, 24 depressed PD patients and 25 healthy controls. Data

72 from 1 non-depressed patient, 3 depressed patients and 2 healthy controls were discarded from

73 analyses for several reasons (see exclusion). Patients were recruited from the Parkinson Centre at the

74 Radboud university medical centre, the Netherlands. Healthy controls were recruited via advertisement,

75 or were partners or acquaintances of patients. Healthy controls and patients were matched for gender,

76 age and IQ measured with the NART (Dutch version of the National Adult Reading Test (Schmand et al.,

77 1991)). Furthermore, patient groups were matched in terms of disease severity (measured with the

78 Unified Parkinson's Disease Rating Scale (UPDRS part III) (Goetz and Stebbins, 2004)) and used similar

79 amounts of dopaminergic medication (LED (Levodopa Equivalent Dose (Esselink et al., 2004))(Table 1).

80 Written informed consent according to the Declaration of Helsinki was obtained from all participants.

81 The study was part of a larger project investigating the neurobiological mechanisms of depression in PD

82 and was approved by the local ethics committee (CMO region Arnhem - Nijmegen, the Netherlands, nr.

$832012 / 43)$.

84 All patients were diagnosed with idiopathic PD according to the UK Brain Bank criteria (Gibb and Lees,

85 1988) by a neurologist specialized in movement disorders (Prof. B.R. Bloem, Dr. R.A. Esselink, Dr. B. Post)

86 and were treated with dopaminergic medication. In the non-depressed patient group 11 patients were

87 treated with levodopa, 2 with dopamine receptor agonists and 9 with both. In the depressed patient

88 group, 14 patients were treated with levodopa, 2 with dopamine receptor agonists and 5 with both.

89 Moreover, 7 depressed patients received antidepressants (paroxetine $n=3$, escitalopram $n=1$,

90 venlafaxine $n=1$ and nortriptyline $n=2$ ). Patients were on stable medication regimes during the course of

91 the study, except for one patient who used duloxetine - a serotonin/noradrenalin reuptake inhibitor 
Timmer e.a.

92 prescribed to treat pain - for 4 weeks between the two testing days (in this case testing days were

93 separated by 17 weeks). The drug was discontinued 4 weeks before the second testing day.

94 Patients were included in the depressed group if they met the DSM-IV criteria for a major $(n=7)$ or minor 95 depressive episode ( $n=12)$, dysthymic disorder $(n=1)$ or adjustment disorder with depressed mood $(n=1)$ within five years before PD diagnosis up until now. This five-year cut-off was chosen because the

97 incidence of depression is significantly higher within the five years before PD diagnosis and therefore likely related to PD pathology (Shiba et al., 2000). Thus, PD patients were selected based on a PD-related depression (history) rather than current depressive symptoms. Seven patients were identified as having current depression. Psychiatric diagnosis was based on structured psychiatric interviews administered during an intake session (MINI-plus (Sheehan et al., 1998)). General exclusion criteria were clinical dementia (Mini Mental State Examination < 24, (Folstein et al., 1975)), psychiatric disorders other than depression (bipolar disorder, schizophrenia, ADHD and drug or alcohol abuse), neurological co-morbidity and hallucinations. Healthy controls were also excluded if they had a history of mood or anxiety disorder, obsessive-compulsive disorder or used any psychotropic medication.

107 and once after abstaining from their dopaminergic medication for at least 18 hours (24 hours for slow 108 release dopamine receptor agonists) (OFF). Patients who used antidepressants were asked to take these 109 antidepressants on both testing days enabling us to assess specifically dopaminergic drug effects on 110 gambling behavior. The order of ON and OFF sessions was counterbalanced in each patient group (Table 111 1). Healthy controls were only tested once. During testing sessions we administered the gambling task 112 described below. Furthermore, on each testing day, participants completed the Beck Depression 113 Inventory (BDI (Beck et al., 1961)) to assess current depressive symptoms. Participants were instructed 114 to answer BDI questions, not according to how they felt over the past week, but according to how they 
Timmer e.a.

115 felt over the past 24 hours, enabling us to assess dopaminergic drug (withdrawal) effects on depression

116 scores. Patients also completed the QUIP rating scale (Weintraub et al., 2012) developed to assess ICD

117 symptoms in PD and were clinically assessed on motor symptom severity (UPDRS part III (Goetz and

118 Stebbins, 2004)).

119 Participants were paid a fixed amount per testing day for participation (healthy controls; 30 Euros, 120 patients; 40 Euros) and received an additional amount of money based on task performance (between $121 \quad$ 2-11 Euros per session).

124 Participants played a well-validated gambling task designed to measure loss aversion (Figure 1) (Tom et 125 al., 2007). During this task, participants were presented with 169 mixed gambles (split into 3 runs) on a computer screen. Each gamble offered a 50/50 percent chance of either gaining or losing varying 127 amounts of money. Potential gains ranged from $+€ 6$ to $+€ 30$ (increments of $€ 2$ ), potential losses ranged

128 from - $€ 3$ to $-€ 15$ (increments of $€ 1$ ). This asymmetric gain-loss range was chosen in order to maximize 129 statistical power, based on the assumption that on average people are twice as sensitive to losses as 130 they are to gains (Tom et al., 2007). Each of the possible gain-loss pairs $(13 \times 13=169)$ was presented once 131 in randomized order. Participants were asked to either accept (play) or reject the gamble by pressing 132 one of two buttons. In order to make participants feel that they were gambling with their own money, 133 and thus avoid "house money effects" (Thaler and Johnson, 1990), endowments at the beginning of this 134 gambling task were earnings from a behavioral experiment immediately preceding the present 135 experiment on the same day. Gambles were not resolved during the experiment to exclude behavioral 136 adjustments on a trial-by-trial basis. However, participants were told to take each gamble seriously, 
bioRxiv preprint doi: https://doi.org/10.1101/069047; this version posted August 11,2016 . The copyright holder for this preprint (which was not certified by peer review) is the author/funder. All rights reserved. No reuse allowed without permission.

Timmer e.a.

137 because at the end of the experiment, 3 gambles would be randomly selected and played for real

138 money.

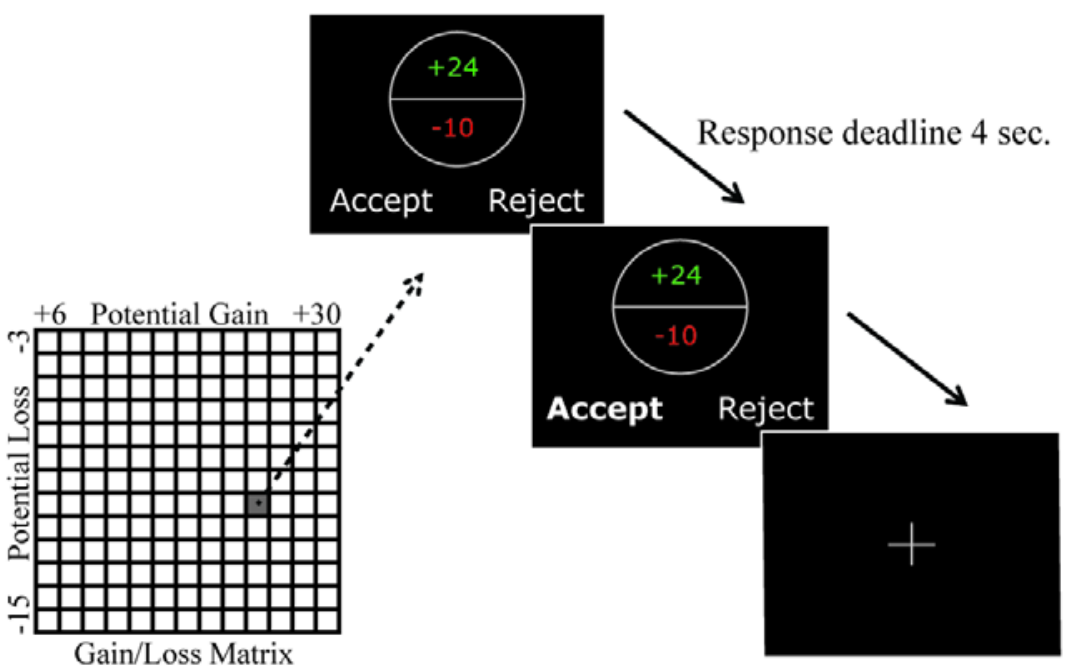

142 Figure 1. Task overview. Participants played a gambling task designed to measure loss aversion. During this task 143 participants were presented with 169 mixed gambles, each offering 50/50 percent change of either gaining or 144 losing varying amounts of money. Gains ranged from $+€ 6$ to $+€ 30$ (increments of $€ 2$ ), losses ranged from - $€ 3$ to $145 € 15$ (increments of €1) (see gain/loss matrix). Each possible gain/loss pair was presented once in randomized 146 order. Participants were asked to either accept (play) or reject the gamble within a maximum time of $4 \mathrm{sec}$. 
Timmer e.a.

152 of choice behavior. One of the most popular accounts of decision making under risk is Prospect Theory

153 (Kahneman and Tversky, 1979). We sought to understand the effects of dopaminergic medication and

154 depression in PD in light of this theory by assessing effects of medication and PD-related depression

155 diagnosis on parameters obtained from a model based on Prospect Theory. Within that framework, the

156 subjective utility of each gamble (SUG) can be approximated by the following equation:

$S U G=$ pGain $*$ Gain - pLoss $*$ Loss $* \lambda$

159 Where $p_{\text {Gain }}$ is the gain probability, $p_{\text {Loss }}$ the loss probability, Gain the gain value of the gamble and Loss the (absolute) loss value of the gamble. The relative weighting of gains and losses is reflected in the loss aversion parameter $\lambda$. If $\lambda>1$, then losses are overvalued relative to gains: a person is loss averse. If $\lambda<1$, then gains are overvalued relative to losses: a person is loss seeking. And if $\lambda=1$, gains and losses are valued equally: a person is gain-loss-neutral.

164 A softmax function was used to estimate the probability of gamble acceptance based on the subjective 165 value of the gamble:

$p($ gamble acceptance $)=\frac{1}{1+e^{-\mu(S U G+c)}}$ constant parameter (c). The inverse temperature parameter reflects consistency of choice behavior. If $\mu$

171 is zero, choices are random, whereas if $\mu$ is highly positive or negative, there is consistency in choice 
Timmer e.a.

172 behavior, with a positive $\mu$ representing higher gamble acceptance with higher gain and lower loss value

173 (and vice versa for negative $\mu$ ). We anticipated $\mu$ to be positive, consistent with a utility maximization

174 strategy, where participants accept more gambles when gain values increase and loss values decrease.

175 The constant parameter (c) reflects a response bias toward or away from gambling irrespective of the

176 value of the gambles. If $c>0$, there is a tendency to accept gambles regardless of their subjective utility.

177 If $c<0$, there is a tendency to reject gambles regardless of their subjective utility.

179 The model that we fitted to the data assumes a linear valuation of gains and losses, in contrast to the curvilinear value function of Prospect Theory. This is a common and reasonable simplifying assumption given the relatively narrow range of gains and losses used in this protocol. We also assumed no

182 subjective transformation of probabilities as described in Prospect Theory and thus assumed equal weights for the 0.5 probability of gains and losses (Tom et al., 2007; De Martino et al., 2010).

\section{Exclusion}

We assessed whether participants' choices were influenced by gain and loss values in an expected manner, i.e. whether participants were utility maximizers (accepting more gambles with increasing gain values and accepting fewer gambles with increasing loss values). Inspection of the individual responses revealed that two participants (one depressed and one non-depressed PD patient) did not meet this a priori assumption, suggesting a lack of understanding of task instructions. In both cases this was during

191 the first testing day. In one case the response graph revealed that the participant accepted more 192 gambles when gain values decreased and loss values increased, thereby unintentionally trying to 193 minimize earnings. During debriefing this participant realized that he had made a mistake. The 194 responses of the other participant were suggestive of random choice behaviour. In both cases, these observations were confirmed by negative temperature parameters $(\mu)$ obtained from the model. These 
Timmer e.a.

two patients were excluded from further analyses. Moreover, two healthy controls were excluded from further analyses because of a lifetime history of depression, while two depressed PD patients were excluded because they failed to finish the study leading to incomplete datasets. The final analysis included 23 healthy controls, 22 non-depressed PD patients and 21 depressed PD patients.

\section{Model fitting and comparison}

We used a hierarchical Bayesian fitting procedure to fit the model to participants' choices as described by Huys et al. (Huys et al., 2011; Huys et al., 2012). This method estimates the mean and the variance of model parameters across all subjects and sessions. These prior parameters then serve to define a normal priori distribution for finding individual values of parameters for each subject and session (i.e. posterior parameters). We hypothesized the a priori distribution of the relevant parameter (i.e. the loss aversion parameter $\lambda$ ) to be different for patients and healthy controls. Therefore we first fitted the model to patient data only. Note that any differences in posterior parameters between patient groups and medication sessions cannot be attributed to parameter regularization employed during fitting, because individual parameters from both patient groups (depressed and non-depressed) and both sessions (ON and OFF medication) were obtained using the same a priori distribution (Huys et al., 2012).

In a subsequent step, to compare PD patients with healthy controls, we fitted the model to healthy control and patient data together (separately for each drug session).

A Bayesian model comparison was conducted to compare the model with 3 parameters $(\lambda, \mu$ and $c)$ with a slightly simpler model, where we forced c to be zero, thereby reducing the number of free parameters.

This model assumed that subjects do not exhibit a response bias toward or away from gambling irrespective of the value of the gambles. A Bayesian model comparison assessed which model best 
Timmer e.a.

219

220

221

222

223

224

225

226

227 where $D^{n}$ is the set of choice data for the $n$th participant, $\theta^{n}$ is the fitted individual parameter for $n$th

228 participant, $\Theta$ and $\Sigma$ are the mean and variance for the group distribution, respectively, $m$ is the

229 number of free parameters of the model, $N$ is the number of participants and $\left|H_{n}\right|$ is the determinant

230 of the Hessian matrix of the log-posterior function at $\theta^{n}$. The first term on the right hand-side of the

231

232

233

234 model with the lowest log-model evidence is the best model.

\section{Statistical analysis}

237 The primary parameter of interest was the loss aversion parameter $(\lambda)$. First we compared depressed PD 238 patients with non-depressed PD patients. Subsequently we compared healthy controls with PD patients

239 (each group and drug session separately). For normally distributed data, we used a mixed ANOVA with 240 drug as within-subject and group as between-subject factor. For non-normally distributed data (Shapiro- 
Timmer e.a.

241 Wilk, $p<0.05)$ we used two-tailed Wilcoxon signed-rank tests to assess within-subject differences and

242 Mann Whitney tests to assess between-group differences. Two-tailed Pearson correlations were used

243 for normally distributed data and two-tailed Spearman correlations for non-normally distributed data.

244 Furthermore, for non-normally distributed data we reported medians and their standard error. Standard

245 errors of the median were computed using Bootstrapping (Efron et al., 1993). By resampling with

246 replacement of the original group sample, we created $10^{5}$ new group samples. The standard error of the

247 median was then defined as the standard deviation of all bootstrapped samples.

\section{Results}

\section{Patient and disease characteristics}

251 Mixed ANOVA of depression scores (BDI) from the PD patients demonstrated a significant group*drug

252 interaction, $F_{(1,41)}=4.19, p=0.047$. Post-hoc paired samples t-test revealed that this interaction was due to

253 a significant drug-induced decrease in depression scores in depressed patients $\left(t_{(20)}=2.19, p=0.041\right)$ but

254 not in non-depressed patients $\left(t_{(21)}=-.60, p=0.56\right)$. There was also a main effect of group, $F_{(1,41)}=17.26$,

$255 p<0.001$, indicating significantly higher depression scores in the depressed patient group. There was no

256 main effect of drug (Figure 2A).

257 Five patients exhibited at least one ICD as assessed with the QUIP rating scale (4 depressed and 1 non-

258 depressed patient) but the proportion of ICD was not different between the two patient groups (Chi ${ }^{2}$

259 test, $p=0.14)$. None of them exhibited pathological gambling. Individual endowments at the beginning of

260 the task varied between participants, as these were earnings from a previous experiment performed on

261 the same day. However, there was no significant main effect of group or drug and no group*drug

262 interaction on these earnings. 
Timmer e.a.

\section{Effects of dopaminergic drugs on loss aversion}

Using Prospect Theory-based analysis, we assessed the computational mechanisms contributing to risky choice. The full model including a constant parameter (c) (reflecting a gambling response bias irrespective of the value of gambles) provided a better account of participants' choices than did a model without this c parameter, indicated by a lower log-model evidence (in patients: 4102 compared with 4374 for the model where (c) was forced to be zero, in healthy controls: 1099 compared with 1131 for the model where (c) was forced to be zero). Therefore, reported results are based on the loss aversion parameter $(\lambda)$ obtained from the full model.

The median loss aversion parameter per group and drug session can be found in Figure 2B. The loss aversion parameter $(\lambda)$ was not normally distributed as indicated by Shapiro-Wilk test. Therefore we used nonparametric statistics. Our analyses revealed a significant group*drug interaction ( $U=149$,

$276 p=0.046)$, which was due to greater drug-induced decreases in loss aversion in depressed patients than

277 in non-depressed patients. If anything, medication increased loss aversion in non-depressed patients.

278 The simple main effects of drug were not significant. There was a near-significant effect of group in the 279 OFF state; depressed patients tended to be more loss-averse than non-depressed patients $(U=151$, $280 p=0.052)$. During the $\mathrm{ON}$ state there was no group effect $(U=215, p=0.70)$. There was no overall main

281 effect of group $(U=191, p=0.33)$ and no overall main effect of drug $(Z=-0.21, p=0.84)$ (Figure $2 B)$. There 282 were no effects of session order. 
Timmer e.a.

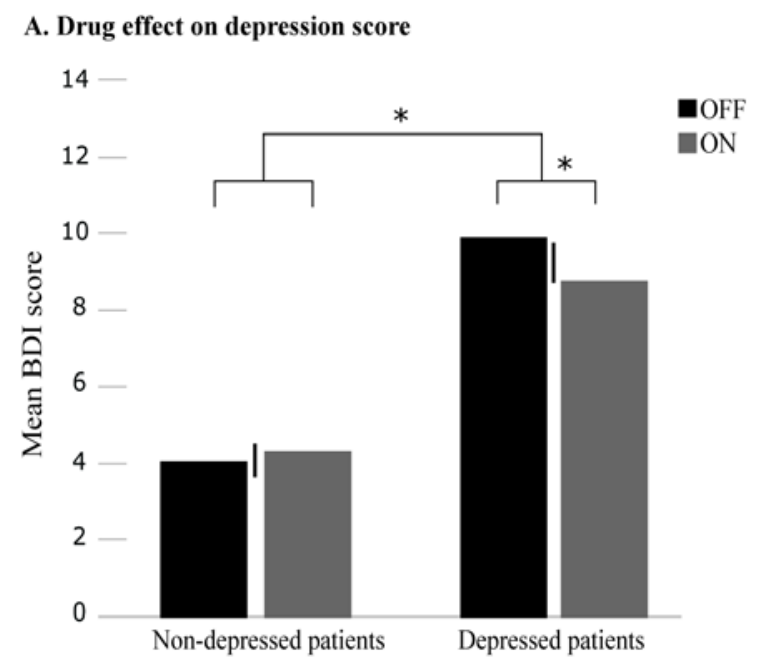

B. Drug effect on loss aversion

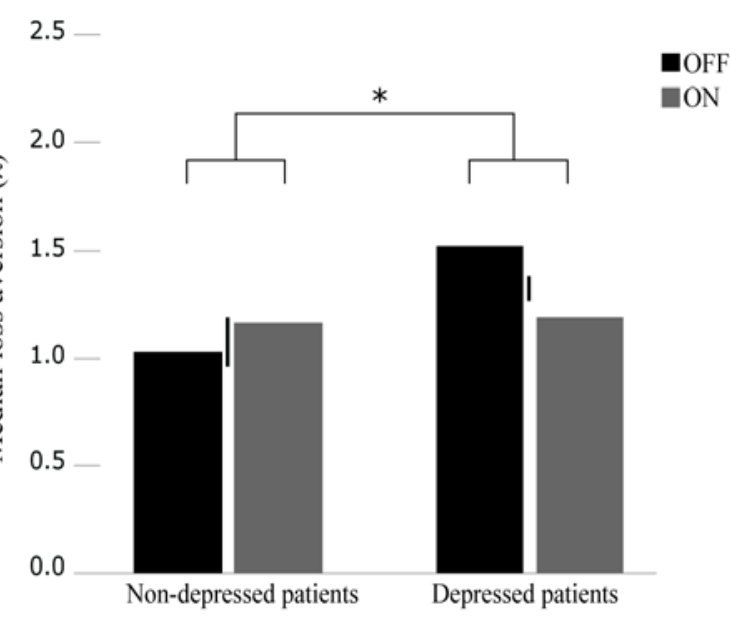

Figure 2. Drug effects per group and drug session. A Mean scores on the Beck Depression Inventory (BDI) per group (depressed and non-depressed patients) and session (OFF session in black, ON session in grey). Error bars represent standard errors of the mean difference. B Median loss aversion parameter $(\lambda)$ per group (depressed and non-depressed patients) and session (OFF session in black, ON session in grey). Error bars represent standard errors of the median difference. ${ }^{*} p<0.05$

To visualize drug and group effects on loss aversion, we plotted, for each group and drug session separately, the degree to which the ratio of rejecting to accepting gambles increased as a function of increases in potential losses (Figure 3). To control for the effects of other factors, such as general drug effects on gambling rate, we plotted the ratio of rejecting to accepting gambles as a function of relative loss differences between pairs of trials, while the effects of different gains were averaged out. A steeper slope indicates greater loss sensitivity. From this Figure 3 it is clear that dopaminergic medication had contrasting effects on loss aversion in depressed and non-depressed PD patients. 
Timmer e.a.

\section{Figure 3. Loss sensitivity}

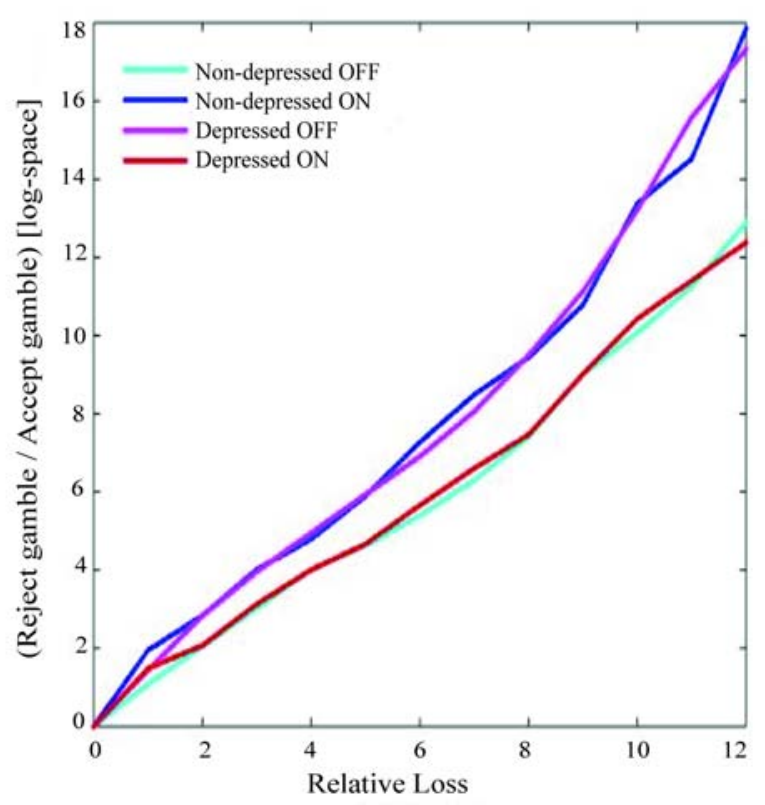

Figure 3. Loss sensitivity. The ratio of the number of rejected gambles divided by the number of accepted gambles per drug session. A steeper slope indicates greater loss sensitivity.

To assess whether drug effects on loss aversion were predicted by current OFF state depression severity, we performed Spearman correlations with BDI scores. Across the whole group there was a significant correlation $\left(r h o_{(41)}=-.348, p=0.022\right)$. This correlation was due to greater drug-induced decreases in loss aversion in patients with higher depression scores (Figure 4A). Additionally, we investigated whether drug effects on depression scores correlated with drug effects on loss aversion. Across the whole group

312 there was a significant correlation $\left(r h o_{(41)}=-.384, p=0.011\right)$, indicating greater drug-induced decreases in 313 loss aversion in patients with greater drug-induced decreases in depression scores. This correlation was 314 strong in depressed patients $\left(r h o_{(19)}=-.592, p=0.005\right)$, but not significant in the non-depressed patients $315\left(r h o_{(20)}=-.021, p=0.93\right)$ and significantly different between groups (Fisher $r-z$ transformation, $z=-2.01$, 
Timmer e.a.

317 (across the two groups, $r h o_{(41)}=0.186, p=0.23$ ).
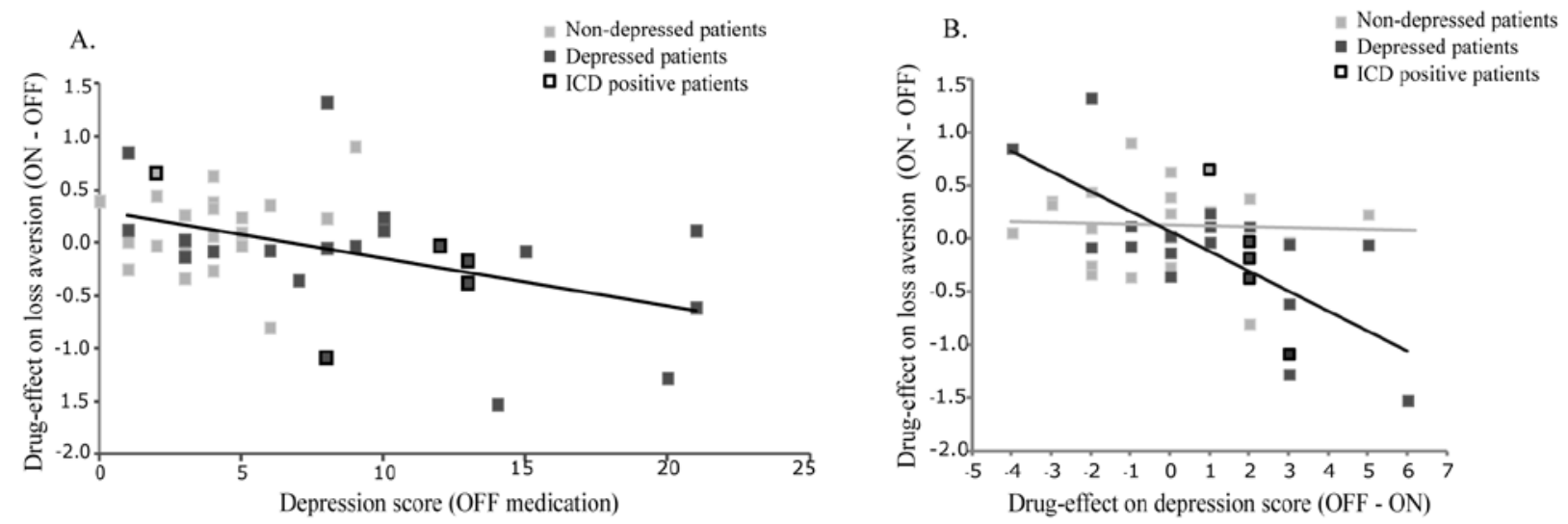

Figure 4. Correlations between (drug effects on) loss aversion and depression. A Correlation between scores on the Beck Depression Inventory during the OFF session (x-axis) and drug effects on loss aversion $(\lambda)$ on the $y$-axis

323 (ON session score minus OFF session score) $\left(r h o_{(41)}=-.384, p=0.011\right)$. B Correlation between drug effects on 324 depression scores on the $\mathrm{x}$-axis (BDI score OFF session minus BDI score ON session) and drug effects on loss 325 aversion $(\lambda)$ on the $y$-axis (ON session score minus OFF session score). Depressed patients are marked in dark grey $326\left(r h o_{(19)}=-.592, p=0.005\right)$, non-depressed patients in light grey $\left(r h o_{(20)}=-.021, p=0.93\right)$. This correlation was 327 significantly different between groups (Fisher $r-z$ transformation, $z=-2.01, p=0.044$ ). Patients who screened positive 328 for having an ICD are marked with a black border. In a supplementary analysis we compared PD patients, each group and drug session separately, with

331 healthy controls. The median loss aversion parameter in healthy controls was significantly higher 332 compared with non-depressed patients OFF medication $(U=150, p=0.019)$, but not different from non- 
Timmer e.a.

depressed patients ON medication and depressed patients during both the ON and OFF session (Table

2).

\section{Gambling response bias and inverse temperature parameter}

The median gambling response bias and inverse temperature parameters are presented in Table 2 per group and drug session. These parameters were not normally distributed as indicated by Shapiro-Wilk test. We therefore used nonparametric statistics. Analyses of the gambling response bias parameter (c) significant group*drug interaction $(U=156, p=0.07)$. There were also no main effects of drug $(Z=-.31$, $p=0.75)$ or group $(U=225, p=0.88)$ and no significant group*drug interaction $(U=226, p=0.90)$ on the

343 inverse temperature parameter.

345 Relative to controls, non-depressed PD patients showed a significantly lower gambling response bias 346 during the OFF session $(U=111, p=0.001)$, but not during the $\mathrm{ON}$ session $(U=177, p=0.08)$. By contrast, 347 depressed PD patients showed a significantly lower gambling response bias during the ON session $348(U=151, p=0.033)$, but not during the OFF session $(U=160, p=0.06)$ relative to controls. There were no 349 differences in terms of the inverse temperature parameter $(\mu)$ between controls and either group of PD 350 patients (ON and OFF medication).

\section{Proportion of accepted gambles}

353 In addition to the computational parameters underlying risky choice, we analyzed the proportion of

354 accepted gambles, which is a compound measure of risky choice. The proportion of accepted gambles in 
Timmer e.a.

depressed PD patients was 53.6\% OFF medication and 57.9\% ON medication. In non-depressed PD patients this was $56.3 \%$ OFF medication and $60.2 \%$ ON medication. The proportion of accepted gambles

357 in healthy controls was $62.2 \%$. Mixed ANOVA in PD patients revealed no significant group*drug 358 interaction $\left(F_{(1,41)}=0.01, p=0.94\right)$ and no main effect of group $\left(F_{(1,41)}=0.31, p=0.58\right)$ or $\operatorname{drug}\left(F_{(1,41)}=2.32\right.$, $359 p=0.136)$. The correlation between drug-induced increases in gamble acceptance and depression scores 360 OFF medication failed to reach significance $\left(r_{(41)}=0.273, p=0.077\right)$. There was also no significant 361 correlation between LED and drug-induced increases in gamble acceptance $\left(r_{(41)}=0.171, p=0.27\right)$. 362 Comparison of patients with healthy controls (each patient group and drug session separately) revealed no significant differences in gamble acceptance.

\section{Discussion}

366 The present study shows that dopaminergic medication induced differential effects on loss aversion

367 during risky choice in PD patients with and without depression. Moreover, we demonstrate that the 368 degree to which medication reduces loss aversion correlates with current depression severity and with 369 drug effects on depression scores: drug-induced reductions in loss aversion were greater in more 370 severely depressed patients and in patients who exhibit greater medication-related decreases in 371 depression scores.

372 It is well known that dopaminergic treatment in PD patients can elicit detrimental side effects in the 373 domain of risky choice. In experimental settings, dopaminergic medication increases risky choice in PD 374 patients (Brand et al., 2004; Euteneuer et al., 2009), while also eliciting abnormal impulsive betting 375 behavior during decision making (Cools et al., 2003). The present findings suggest that these effects 376 might have been driven by patients with relatively higher depression scores in the OFF medication state.

377 Critically, in those prior studies, the computational mechanisms underlying increased risky choice were 
Timmer e.a.

not investigated. In this study, we adopted a computational approach, enabling us to isolate the mechanisms underlying drug-induced change during risky choice in (specific subgroups of) PD patients.

In line with results from a number of clinical trials in PD, we observed that dopaminergic medication significantly decreased depression scores in the depressed PD group (Barone et al., 2006; Barone et al., 2010; Stacy et al., 2010). Our data also revealed that dopamine-induced changes in depression scores correlated with dopamine-induced changes in loss aversion. Patients with the greatest antidepressant effect of dopaminergic medication also exhibited the greatest decrease in loss aversion. These findings raise the hypothesis that dopamine-induced changes in loss aversion might underlie the beneficial effects of dopaminergic medication on depressive symptoms in PD.

In prior work, we have put forward the dopamine overdose hypothesis to account for the detrimental effects of dopaminergic medication on punishment-based learning and decision-making. This hypothesis states that dopaminergic medication doses necessary to remedy dopamine levels in severely depleted dorsal striatum might detrimentally overdose dopamine levels in the relatively intact ventral striatum (Cools et al., 2001, 2003; Cools, 2006). According to this hypothesis, one might expect that any abnormal decrease in loss aversion is seen only in non-depressed PD patients with a putatively intact ventral striatum, while not extending to depressed PD patients, who have been argued to exhibit ventral striatal dopamine deficiency (Vriend et al., 2014). By contrast, the current study suggests that depressed PD patients are particularly at risk for developing detrimental effects of dopaminergic medication on risky choice. This finding concurs generally with clinical evidence indicating that PD patients who exhibit more severe depressive symptoms are at increased risk for having ICD (Pontone et al., 2006; Isaias et al., 2008; Voon et al., 2011b), although a strong link between (dopamine-induced decreases in) loss aversion and ICD has yet to be established (Voon et al., 2011a; Giorgetta et al., 2014). 
Timmer e.a.

400

401

402

403

404

405

406

407

408

409

410

411

412

413

414

415

416

417

418

419

420

421

422

423

Neuroimaging studies with healthy and depressed individuals (without PD) have revealed neural loss aversion in several limbic brain regions, including the striatum (Tom et al., 2007; Canessa et al., 2013; Chandrasekhar Pammi et al., 2015). Moreover, evidence from work in healthy volunteers, PD patients and rodents indicates that both drug-induced impulsivity and risky choice as well as depression are accompanied by low striatal dopamine D2 receptor and dopamine transporter (DAT) availability (Remy et al., 2005; Weintraub et al., 2005; Boileau et al., 2009; Buckholtz et al., 2010; Cocker et al., 2012; Norbury et al., 2013; Vriend et al., 2013). Together these observations raise the hypothesis that druginduced changes in loss aversion in PD could reflect impaired auto-regulation of striatal dopamine levels. This hypothesis might be tested in future studies by combining the use of neuroeconomic tools and controlled medication withdrawal in PD, with neurochemical imaging of dopamine D2 receptor availability and dopamine release.

A number of limitations of the current study should be highlighted. First, in contrast to recent findings from (Chandrasekhar Pammi et al., 2015), who observed greater loss aversion in depressed patients (without PD), we did not observe significantly greater loss aversion in our depressed PD patients OFF (or ON) medication than controls. This might reflect the fact that not all our patients were currently depressed. Instead we included patients based on the presence of a history of depression. This should be tested in future work with a larger sample of currently depressed patients. Second, the design was not optimized for comparing patients with controls, who were tested only once OFF medication, in contrast to the patients, who were tested twice, once ON and once OFF medication. Moreover, our control group exhibited a median loss aversion parameter of 1.4 , which is relatively low compared with previous studies with healthy individuals in which loss aversion estimates ranged between 1.4 and 2.0 (Tom et al., 2007; Sokol-Hessner et al., 2009; De Martino et al., 2010). This apparent discrepancy with prior work might reflect the fact that our control group was somewhat older, but in any case caution is warranted when interpreting the results from the comparisons with healthy controls. Finally, the 
Timmer e.a.

424 pattern of medication effects in terms of the proportion of accepted gambles, which did not exhibit a

425 significant drug*group effect, is quite different from that in terms of the loss aversion parameter, which

426 did exhibit a significant drug*group effect. This is not surprising because our model takes into account

427 the prior theoretical insight that the proportion of accepted gambles is a function of multiple

428 parameters, including not just loss aversion, but also gambling response bias. In fact, effects of

429 medication and depression on loss aversion were isolated, precisely because we disentangled it from

430 any (non significant) variability in terms of gambling response bias. As such, the discrepancy between

431 pattern of effects on the proportion of accepted gambles and that on loss aversion highlights the

432 strength of the adopted modeling approach. 
Timmer e.a.

\section{References}

448 Barone P, Poewe W, Albrecht S, Debieuvre C, Massey D, Rascol O, Tolosa E, Weintraub D (2010) randomised, double-blind, placebo-controlled trial. Lancet Neurol 9:573-580.

Barone P, Scarzella L, Marconi R, Antonini A, Morgante L, Bracco F, Zappia M, Musch B, Depression Parkinson Italian S (2006) Pramipexole versus sertraline in the treatment of depression in Parkinson's disease - A national multicenter parallel-group randomized study. J Neurol 253:601-

Beck AT, Erbaugh J, Ward CH, Mock J, Mendelsohn M (1961) AN INVENTORY FOR MEASURING DEPRESSION. Arch Gen Psychiatry 4:561-\&.

Boileau I, Guttman M, Rusjan P, Adams JR, Houle S, Tong J, Hornykiewicz O, Furukawa Y, Wilson AA, Kapur S, Kish SJ (2009) Decreased binding of the D-3 dopamine receptor-preferring ligand C-11 ()-PHNO in drug-nave Parkinsons disease. Brain 132:1366-1375.

Brand M, Labudda K, Kalbe E, Hilker R, Emmans D, Fuchs G, Kessler J, Markowitsch HJ (2004) Decisionmaking impairments in patients with Parkinson's disease. Behav Neurol 15:77-85.

Buckholtz JW, Treadway MT, Cowan RL, Woodward ND, Li R, Ansari MS, Baldwin RM, Schwartzman AN, Shelby ES, Smith CE, Kessler RM, Zald DH (2010) Dopaminergic Network Differences in Human

Canessa N, Crespi C, Motterlini M, Baud-Bovy G, Chierchia G, Pantaleo G, Tettamanti M, Cappa SF (2013) The Functional and Structural Neural Basis of Individual Differences in Loss Aversion. Journal of Neuroscience 33:14307-+. 
Timmer e.a.

patients and healthy individuals: A functional MRI investigation. The neuroradiology journal 28:97-105.

Cocker PJ, Dinelle K, Kornelson R, Sossi V, Winstanley CA (2012) Irrational Choice under Uncertainty Correlates with Lower Striatal D-2/3 Receptor Binding in Rats. Journal of Neuroscience 32:15450-15457.

Cools R (2006) Dopaminergic modulation of cognitive function-implications for L-DOPA treatment in Parkinson's disease. Neurosci Biobehav Rev 30:1-23.

Cools R, Altamirano L, D'Esposito M (2006) Reversal learning in Parkinson's disease depends on medication status and outcome valence. Neuropsychologia 44:1663-1673.

Cools R, Barker RA, Sahakian BJ, Robbins TW (2001) Enhanced or impaired cognitive function in Parkinson's disease as a function of dopaminergic medication and task demands. Cerebral Cortex 11:1136-1143.

Cools R, Barker RA, Sahakian BJ, Robbins TW (2003) L-Dopa medication remediates cognitive inflexibility, but increases impulsivity in patients with Parkinson's disease. J Psychopharmacol 17:A57-A57.

De Martino B, Camerer CF, Adolphs R (2010) Amygdala damage eliminates monetary loss aversion. Proceedings of the National Academy of Sciences of the United States of America 107:37883792.

Efron B, Tibshirani RJ (1993). An Introduction to the Bootstrap. 1st ed. New York: Chapman and Hall/CRC.

Eshel N, Roiser JP (2010) Reward and Punishment Processing in Depression. Biological Psychiatry 68:118124.

Esselink RAJ, de Bie RMA, de Haan RJ, Lenders M, Nijssen PCG, Staal MJ, Smeding HMM, Schuurman PR, Bosch DA, Speelman JD (2004) Unilateral pallidotomy versus bilateral subthalamic nucleus stimulation in PD - A randomized trial. Neurology 62:201-207. 
Timmer e.a.

494

495

496

497

498

499

500

501

502

503

504

505

506

507

508

509

510

Euteneuer F, Schaefer F, Stuermer R, Boucsein W, Timmermann L, Barbe MT, Ebersbach G, Otto J, Kessler J, Kalbe E (2009) Dissociation of decision-making under ambiguity and decision-making under risk in patients with Parkinson's disease: A neuropsychological and psychophysiological study. Neuropsychologia 47:2882-2890.

Folstein MF, Folstein SE, McHugh PR (1975) MINI-MENTAL STATE - PRACTICAL METHOD FOR GRADING COGNITIVE STATE OF PATIENTS FOR CLINICIAN. Journal of Psychiatric Research 12:189-198.

Frank MJ, Seeberger LC, O'Reilly RC (2004) By carrot or by stick: Cognitive reinforcement learning in Parkinsonism. Science 306:1940-1943.

Gibb WRG, Lees AJ (1988) THE RELEVANCE OF THE LEWY BODY TO THE PATHOGENESIS OF IDIOPATHIC PARKINSONS-DISEASE. J Neurol Neurosurg Psychiatry 51:745-752.

Giorgetta C, Grecucci A, Rattin A, Guerreschi C, Sanfey AG, Bonini N (2014) To play or not to play: A personal dilemma in pathological gambling. Psychiatry Res 219:562-569.

Goetz CG, Stebbins GT (2004) Assuring interrater reliability for the UPDRS motor section: Utility of the UPDRS teaching tape. Mov Disord 19:1453-1456.

Gotham AM, Brown RG, Marsden CD (1988) FRONTAL COGNITIVE FUNCTION IN PATIENTS WITH PARKINSONS-DISEASE ON AND OFF LEVODOPA. Brain 111:299-321.

Gradin VB, Kumar P, Waiter G, Ahearn T, Stickle C, Milders M, Reid I, Hall J, Steele JD (2011) Expected value and prediction error abnormalities in depression and schizophrenia. Brain 134:1751-1764.

Huys QJM, Eshel N, O'Nions E, Sheridan L, Dayan P, Roiser JP (2012) Bonsai Trees in Your Head: How the Pavlovian System Sculpts Goal-Directed Choices by Pruning Decision Trees. Plos Computational Biology 8.

Huys QJM, Cools R, Goelzer M, Friedel E, Heinz A, Dolan RJ, Dayan P (2011) Disentangling the Roles of Approach, Activation and Valence in Instrumental and Pavlovian Responding. Plos Computational Biology 7. 
Timmer e.a.

518

519

520

521

522

523

524

525

526

527

528

529

530

531

532

533

534

535

536

537

538

539

540

541

Isaias LU, Siri C, Cilia R, De Gaspari D, Pezzoli G, Antonini A (2008) The relationship between impulsivity and impulse control disorders in Parkinson's disease. Mov Disord 23:411-415.

Joutsa J, Martikainen K, Vahlberg T, Voon V, Kaasinen V (2012) Impulse control disorders and depression in Finnish patients with Parkinson's disease. Parkinsonism \& Related Disorders 18:155-160.

Kahneman D, Tversky A (1979) Prospect Theory - Analysis of Decision under Risk. Econometrica 47:263291.

Kahneman D, Tversky A (1984) CHOICES, VALUES, AND FRAMES. American Psychologist 39:341-350.

Kass RE, Raftery AE (1995) BAYES FACTORS. Journal of the American Statistical Association 90:773-795.

MacKay DJC (2003). Information theory, inference, and learning algorithms. Cambridge: Cambridge University.

Maddox WT, Gorlick MA, Worthy DA, Beevers CG (2012) Depressive symptoms enhance lossminimization, but attenuate gain-maximization in history-dependent decision-making. Cognition 125:118-124.

Maricle RA, Valentine RJ, Carter J, Nutt JG (1998) Mood response to levodopa infusion in early Parkinson's disease. Neurology 50:1890-1892.

Norbury A, Manohar S, Rogers RD, Husain M (2013) Dopamine Modulates Risk-Taking as a Function of Baseline Sensation-Seeking Trait. Journal of Neuroscience 33:12982-12986.

Piray P, Zeighami Y, Bahrami F, Eissa AM, Hewedi DH, Moustafa AA (2014) Impulse Control Disorders in Parkinson's Disease Are Associated with Dysfunction in Stimulus Valuation But Not Action Valuation. Journal of Neuroscience 34:7814-7824.

Pontone G, Williams JR, Bassett SS, Marsh L (2006) Clinical features associated with impulse control disorders in Parkinson disease. Neurology 67:1258-1261.

Remy P, Doder M, Lees A, Turjanski N, Brooks D (2005) Depression in Parkinson's disease: loss of dopamine and noradrenaline innervation in the limbic system. Brain 128:1314-1322. 
Timmer e.a.

542 543

544

545

546

547

548

549

550

551

552

553

554

555

556

557

558

559

560

561

562

563

564

565

Robinson OJ, Cools R, Carlisi CO, Sahakian BJ, Drevets WC (2011) Ventral Striatum Response During Reward and Punishment Reversal Learning in Unmedicated Major Depressive Disorder. Am J Psychiatry.

Schmand B, Bakker D, Saan R, Louman J (1991) The Dutch Reading Test for Adults: a measure of premorbid intelligence level. Tijdschrift voor gerontologie en geriatrie 22:15-19.

Schrag A (2006) Quality of life and depression in Parkinson's disease. Journal of the Neurological Sciences 248:151-157.

Seppi K, Weintraub D, Coelho M, Perez-Lloret S, Fox SH, Katzenschlager R, Hametner E-M, Poewe W, Rascol O, Goetz CG, Sampaio C (2011) The Movement Disorder Society Evidence-Based Medicine Review Update: Treatments for the Non-Motor Symptoms of Parkinson's Disease. Mov Disord 26:S42-S80.

Sheehan DV, Lecrubier Y, Sheehan KH, Amorim P, Janavs J, Weiller E, Hergueta T, Baker R, Dunbar GC (1998) The Mini-International Neuropsychiatric Interview (MINI): The development and validation of a structured diagnostic psychiatric interview for DSM-IV and ICD-10. J Clin Psychiatry 59:22-33.

Shiba M, Bower JH, Maraganore DM, McDonnell SK, Peterson BJ, Ahlskog JE, Schaid DJ, Rocca WA (2000) Anxiety disorders and depressive disorders preceding Parkinson's disease: A case-control study. Mov Disord 15:669-677.

Sokol-Hessner P, Hsu M, Curley NG, Delgado MR, Camerer CF, Phelps EA (2009) Thinking like a trader selectively reduces individuals' loss aversion. Proceedings of the National Academy of Sciences of the United States of America 106:5035-5040.

Stacy MA, Murck H, Kroenke K (2010) Responsiveness of motor and nonmotor symptoms of Parkinson disease to dopaminergic therapy. Progress in Neuro-Psychopharmacology \& Biological Psychiatry 34:57-61. 
Timmer e.a.

Swainson R, Rogers RD, Sahakian BJ, Summers BA, Polkey CE, Robbins TW (2000) Probabilistic learning and reversal deficits in patients with Parkinson's disease or frontal or temporal lobe lesions: possible adverse effects of dopaminergic medication. Neuropsychologia 38:596-612.

Thaler RH, Johnson EJ (1990) GAMBLING WITH THE HOUSE MONEY AND TRYING TO BREAK EVEN - THE EFFECTS OF PRIOR OUTCOMES ON RISKY CHOICE. Management Science 36:643-660.

Tom SM, Fox CR, Trepel C, Poldrack RA (2007) The neural basis of loss aversion in decision-making under risk. Science 315:515-518.

van der Schaaf ME, van Schouwenburg MR, Geurts DE, Schellekens AF, Buitelaar JK, Verkes RJ, Cools R (2014) Establishing the dopamine dependency of human striatal signals during reward and punishment reversal learning. Cereb Cortex 24:633-642.

Voon V, Gao J, Brezing C, Symmonds M, Ekanayake V, Fernandez H, Dolan RJ, Hallett M (2011a) Dopamine agonists and risk: impulse control disorders in Parkinson's; disease. Brain 134:14381446.

Voon V, Sohr M, Lang AE, Potenza MN, Siderowf AD, Whetteckey J, Weintraub D, Wunderlich GR, Stacy M (2011b) Impulse Control Disorders in Parkinson Disease: A Multicenter Case-Control Study. Annals of Neurology 69:986-996.

Vriend C, Pattij T, van der Werf YD, Voorn P, Booij J, Rutten S, Berendse HW, van den Heuvel OA (2014) Depression and impulse control disorders in Parkinson's disease: Two sides of the same coin? Neurosci Biobehav Rev 38:60-71.

Vriend C, Raijmakers P, Veltman DJ, van Dijk KD, van der Werf YD, Foncke EM, Smit JH, Berendse HW, van den Heuvel OA (2013) Depressive symptoms in Parkinson's disease are related to reduced [123I]FP-CIT binding in the caudate nucleus. J Neurol Neurosurg Psychiatry.

Weintraub D, Mamikonyan E, Papay K, Shea JA, Xie SX, Siderowf A (2012) Questionnaire for impulsivecompulsive disorders in Parkinson's Disease-Rating Scale. Mov Disord 27:242-247. 
Timmer e.a.

590

591

592

593

594

595

596

597

598

599

600

601

602

603

604

605

606

607

608

609

610

611

612
Weintraub D, Koester J, Potenza MN, Siderowf AD, Stacy M, Voon V, Whetteckey J, Wunderlich GR, Lang AE (2010) Impulse Control Disorders in Parkinson Disease A Cross-Sectional Study of 3090 Patients. Archives of Neurology 67:589-595.

Weintraub D, Newberg AB, Cary MS, Siderowf AD, Moberg PJ, Kleiner-Fisman G, Duda JE, Stern MB, Mozley D, Katz IR (2005) Striatal dopamine transporter imaging correlates with anxiety and depression symptoms in Parkinson's disease. J Nucl Med 46:227-232. 
Timmer e.a.

613 Table 1. Group characteristics

\begin{tabular}{|c|c|c|c|}
\hline & Depressed PD & Non-depressed PD & Healthy controls \\
\hline & $n=21$ & $\mathrm{n}=22$ & $n=23$ \\
\hline Gender, men & 13 & 13 & 14 \\
\hline Age, years & $58.5(5.8)$ & $61.0(7.6)$ & $60.9(5.9)$ \\
\hline NART-IQ & $96.2(11.6)$ & $97.0(15.5)$ & $100.7(13.7)$ \\
\hline MMSE & $28.5(1.4)$ & $28.6(1.3)$ & $28.8(1.2)$ \\
\hline Hoehn \& Yahr & $1.6(0.4)$ & $1.8(0.5)$ & - \\
\hline UPDRS - III (OFF) & $22.7(9.6)$ & $22.2(6.5)$ & - \\
\hline Disease duration, years & $5.1(3.5)$ & $4.5(2.2)$ & - \\
\hline LED mg/day & $551(248)$ & $627(275)$ & - \\
\hline LED agonists $\mathrm{mg} /$ day & $71(122)$ & 103 (129) & - \\
\hline BDI (OFF) & $9.9(6.1)$ & $4.0(2.3)$ & $3.1(2.1)$ \\
\hline Current ICD & 4 & 1 & - \\
\hline First session ON & 11 & 9 & - \\
\hline Days between sessions & $23(27)$ & $21(20)$ & - \\
\hline
\end{tabular}

614 Values represent numbers or mean (standard deviation)

615

616

617

618

619 
Timmer e.a.

Table 2. Model parameters per group and drug session

\begin{tabular}{lll}
\hline & OFF session & ON session \\
\hline Loss aversion $(\boldsymbol{\lambda})$ & $1.51(3.0)$ & $1.19(2.7)$ \\
Depressed patients & $1.01(3.2)$ & $1.16(2.6)$ \\
Non-depressed patients & $1.37(2.8)$ & - \\
Healthy controls & & $-1.30(13.8)$ \\
Gambling response bias (c) & $-1.73(14.9)$ & $-1.05(8.9)$ \\
Depressed patients & $-2.71(9.4)$ & - \\
Non-depressed patients & $-0.65(11.1)$ & - \\
Healthy controls & & $0.94(1.9)$ \\
Inverse temperature $(\boldsymbol{\mu})$ & $0.93(2.1)$ & $1.09(2.2)$ \\
Depressed patients & $0.89(1.5)$ & - \\
Non-depressed patients & $1.06(2.1)$ & \\
\hline Healthy controls & & \\
\hline Values represent median & & \\
\hline
\end{tabular}

